\title{
Risk Factors and Antibiotic Sensitivity Pattern of Staphylococcus aureus Isolates from Suppurative Lesions at a Tertiary Care Hospital
}

\author{
T. Susitha ${ }^{1}$ and M. Sudha ${ }^{2}$ * \\ ${ }^{1}$ Department of Microbiology, Kanyakumari Government Medical College, \\ Tamil Nadu, India \\ ${ }^{2}$ Department of Microbiology, Government Theni Medical College, Tamil Nadu, India \\ *Corresponding author
}

\begin{tabular}{|l|}
\hline Ke y w o r d s \\
Methicillin \\
resistance, \\
Staphylococcus \\
aureus, Risk \\
factors, Antibiotic \\
sensitivity
\end{tabular}

\section{Introduction}

The cluster-forming micro-organism, Staphylococcus aureus (S. aureus) is usually associated with a number of human diseases, which involves intense suppuration and necrosis of tissue. The high prevalence of $S$.

\section{A B S T R A C T}

The early detection of Methicillin resistance along with its risk factors is of prime importance in the prognosis of $S$. aureus infections. The marked difference between the antibiogram of MRSA and MSSA isolates creates a difficult scenario in initiating treatment for $S$. aureus infections. The study aims to identify the risk factors and antibiotic sensitivity pattern of MRSA from pus samples. The present study was conducted at the Department of Microbiology, Kanyakumari Medical College from September 2015 to August 2016 from 100 non-duplicate $S$. aureus isolates from pus samples. The $S$. aureus isolates were confirmed by standard methods and methicillin resistance detected by Cefoxitin $(30 \mu \mathrm{g})$ disc. The antibiotic sensitivity pattern was interpreted as per CLSI guidelines. Among the 34 MRSA isolates, 25 were from males and 9 from females of which majority $9(36 \%)$ were from boys of $\leq 15$ years of age. Among the risk factors associated with MRSA, surgery had accounted for $26.5 \%$ and diabetes $11.8 \%$. Resistance to Erythromycin, Gentamicin and Ciprofloxacin was increasingly noted among MRSA isolates and all of them were sensitive to Vancomycin, Teicoplanin, Tigecycline and Linezolid. The early detection of Methicillin resistance among $S$. aureus together with timely and ideal antibiotic initiation helped by antibiotic sensitivity testing is the need of the hour, which limits the spread of MRSA infections in hospital setting. The role played by a clinical microbiologist in early detection of MRSA is very critical which helps to avoid multidrug resistance, in an era of very limited antibiotics. 
prognosis of $S$. aureus infections, since a steady rise of them is being noted.

Emergence of Methicillin Resistance $S$. aureus (MRSA) is attributed to multiple factors like longer duration of stay in hospital, admission in an intensive care unit, prolonged antimicrobial therapy, surgical procedures, device exposure (urinary catheter, central venous catheter) and even close proximity to a patient in the hospital who is infected or colonized with MRSA ${ }^{1}$. Infections by MRSA are more difficult to be dealt than those caused by Methicillin-Sensitive $S$. aureus (MSSA) isolates ${ }^{2}$. The marked difference between the antibiogram of MRSA and MSSA isolates creates a difficult scenario in initiating treatment for $S$. aureus infections. MRSA easily spreads among patients in hospital adding to the problem.

The multidrug resistance of MRSA isolates is of alarming problem paving way for costlier treatment regimens. ${ }^{3}$ Effort for manufacturing newer antibiotic for Gram positive infections remains uncertain and only fewer antimicrobials remains as alternatives for them. MRSA strains are resistant to all betalactam agents, and often to other drugs such as aminoglycosides and fluoroquinolones ${ }^{2}$. At present, Glycopeptides and Linezolid are the only available agents of choice in the treatment of systemic infections with MRSA. The study aims to assess the risk factors for MRSA and their drug susceptibility pattern from pus specimens at a tertiary care teaching hospital in South Tamil Nadu.

\section{Materials and Methods}

The present study was conducted at the Department of Microbiology, Kanyakumari Government Medical College from September 2015 to August 2016 from 100 non-duplicate $S$. aureus isolates from pus samples. Informed written consent and a filled in proforma were obtained from the participants involved in the study. The $S$. aureus isolates were confirmed by standard methods and methicillin resistance detected by disc diffusion on Mueller-Hinton agar by Cefoxitin $(30 \mu \mathrm{g})$ disc.

The following antibiotic discs were used to determine the sensitivity of $S$. aureus isolates-Penicillin (10 IU), Erythromycin $(15 \mu \mathrm{g}), \quad$ Clindamycin $(2 \mu \mathrm{g}), \quad$ Gentamicin

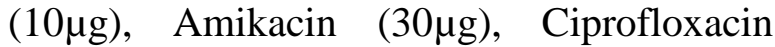
$(5 \mu \mathrm{g}), \quad$ Clotrimoxazole $\quad(1.25 / 23.75 \mu \mathrm{g})$, Vancomycin $(30 \mu \mathrm{g})$, Teicoplanin $(30 \mu \mathrm{g})$, Tigecycline $(15 \mu \mathrm{g})$ and Linezolid $(30 \mu \mathrm{g})$ (HiMedia Laboratories, Mumbai, India). The drug sensitivity for the isolates was interpreted as per CLSI guidelines ${ }^{4}$. ATCC 25923 S. aureus strain was included for each and every procedure as quality control.

\section{Data analysis}

Data regarding the subjects were described in terms of percentages. The ages of the subjects were compared between the genders by student's unpaired ' $t$ ' test. The sensitive, resistant and intermediately susceptible were described in terms of percentages. The statistical procedures were performed with the help of the statistical software IBM SPSS statistics 20 . The $\mathrm{p}$ value less than 0.05 was considered as significant $(\mathrm{p}<0.05)$ in two tailed test.

\section{Results and Discussion}

During the one year period, 100 S. aureus isolates were screened for methicillin resistance by Cefoxitin disc diffusion method from pus samples. A total of 34 isolates showed resistance to methicillin while the remaining 66 of them were methicillin sensitive. Among the MRSA isolates, 25 were from males and 9 from females of which majority 9 (36\%) were from boys of $\leq 15$ years of age (Figure 1). The mean age of males was 
30.7 years and that of females 39.2 years among MRSA isolates and was not found significant $(\mathrm{p}>0.05)$.

A total of $24(75 \%)$ and eight $(25 \%)$ of the MRSA isolates were from patients with less than two weeks stay in hospital and more than two weeks respectively. Figure 2 depicts the categorization of MRSA isolates on infection basis. Most of the MRSA isolates are from Surgery department 11(32.3\%) followed by Pediatrics 7(20.6\%), Orthopedics 4(11.8\%), Obstetrics and Gynecology 3(8.8\%), Dermatology $3(8.8 \%) \quad$ ENT $1(2.9)$, Ophthalmology $1(2.9 \%)$ and an isolate (2.9\%) from Neurosurgery department.

Table 1 shows the risk factors associated with MRSA where surgery had accounted for $26.5 \%$ and diabetes $11.8 \%$. Marked differences in antibiotic sensitivity pattern of the MSSA and MRSA isolates were observed (Table 2). Resistance to Erythromycin, Gentamicin and Ciprofloxacin was increasingly noted among MRSA isolates and all of them were sensitive to Vancomycin, Teicoplanin, Tigecycline and Linezolid.

The increasing prevalence of MRSA among clinical specimens, conditions the patient for prolonged treatment and the associated risk factors adds to the problem. The easily available and standard Cefoxitin disc diffusion method picks up Methicillin resistant isolates from the specimens. Difficulty in deciding the initial antibiotic exists for $S$. aureus and so it is wise to perform antibiotic sensitivity testing for all $S$. aureus isolates before initiating treatment.

In this study, majority of the MRSA isolates were from males $(25 \%)$ while the remaining were females $(9 \%)$. This is similar to the study by Thangavel et al., ${ }^{5}$, where $30 \%$ of the MRSA isolates were from males while $10 \%$ from females. On the contrary, the study from Doon valley hospitals ${ }^{6}$, showed that most of the MRSA isolates were from females $60.86 \%$ while males were $39.13 \%$ only. The study by Buzaid et al., ${ }^{7}$, found no significant difference between males 28 (31.8\%) and females 34 (30.4\%) among MRSA isolates.

Majority of the MRSA isolates were from the Surgery department (32.3\%), followed by Paediatrics (20.6\%), Orthopaedics (11.8\%), and Dermatology (8.8\%) in the present study. The study by Arora et al., ${ }^{8}$, too had found highest prevalence from surgical units $(54.8 \%)$. Surgery was definitely an associated factor in MRSA infections as those patients are bound to take antimicrobials for long duration. Sarma et al., ${ }^{9}$, had found that MRSA was associated with $34 \%$ of infections in Orthopaedics and $18 \%$ in surgical units but only $1 \%$ in medical units. The highest percentages of isolates were from Intensive Care Units (34\%) in the study by Sadaka et al. ${ }^{10}$.

In this study, almost most of the MRSA were isolated from wound infection $(29.4 \%)$ while surgical site infections constituted $26.5 \%$. The remaining isolates were from boil/furuncle (20.6\%), abscess (14.7\%) and burns (2.9\%). This is comparable to the study by Terry Ali $e t$ al. ${ }^{11}$, where wound infection (21.4\%), isolated majority of the MRSA isolates. The study from Pondicherry ${ }^{12}$, found a high prevalence from post-operative surgical infections (80\%). Frazee et al., ${ }^{13}$, found that MRSA, was strongly associated with infection typefuruncle. Most of the MRSA isolates were from patients who had undergone surgery $(26.5 \%)$, while $11.8 \%$ isolates with diabetes, $2.9 \%$ with burns, $2.9 \%$ with HIV and $2.9 \%$ with Job's syndrome in the present study. Surgical site infections, both superficial and deep, could be caused by MRSA. Repeated surgeries and hence prolonged stay, makes way for MRSA infections. Among diabetics, 
the decrease in the neutrophil activity plays a predominant role for MRSA infection. But the study from New Delhi ${ }^{14}$ found a $51.6 \%$ association with high risk patients like burns. This was attributed to the longer stay in hospital and the usage of multiple antibiotics.

In this study, duration of stay at hospital was not a risk factor for MRSA infection. Similarly, the study from Northeastern India ${ }^{9}$ had found that duration of hospital stay had no significant association with MRSA infection. But Mehta et al., ${ }^{15}$, observed significant association of MRSA and hospital stay of more than 15 days duration. Regarding Macrolides, Erythromycin resistance seems to be on rise among MRSA isolates. In the present study, resistance was noted among 5 (7.6\%) of the MSSA isolates while it was 21 (61.8\%) among MRSA isolates. Arora et al., ${ }^{8}$, found that $61.7 \%$ of MRSA isolates were resistant to this drug. Frazee et al., ${ }^{13}$, and Rao et al., ${ }^{16}$, found $56.8 \%$ and $45-48 \%$ resistance among MRSA isolates respectively. This is in contrast to the study from Coimbatore ${ }^{17}$, where the Erythromycin resistance was $20.5 \%$ only.

Table.1 MRSA and risk factors

\begin{tabular}{|l|l|c|}
\hline \multirow{2}{*}{ Risk factors } & \multicolumn{2}{|c|}{ MRSA } \\
\cline { 2 - 3 } & No & \% \\
\hline Surgery & 9 & 26.5 \\
\hline Diabetes & 4 & 11.8 \\
\hline Burns & 1 & 2.9 \\
\hline Job's syndrome & 1 & 2.9 \\
\hline HIV & 1 & 2.9 \\
\hline Unidentified & 18 & 52.9 \\
\hline Total & 34 & 100 \\
\hline
\end{tabular}

Table.2 Antibiotic sensitivity pattern of $S$. aureus

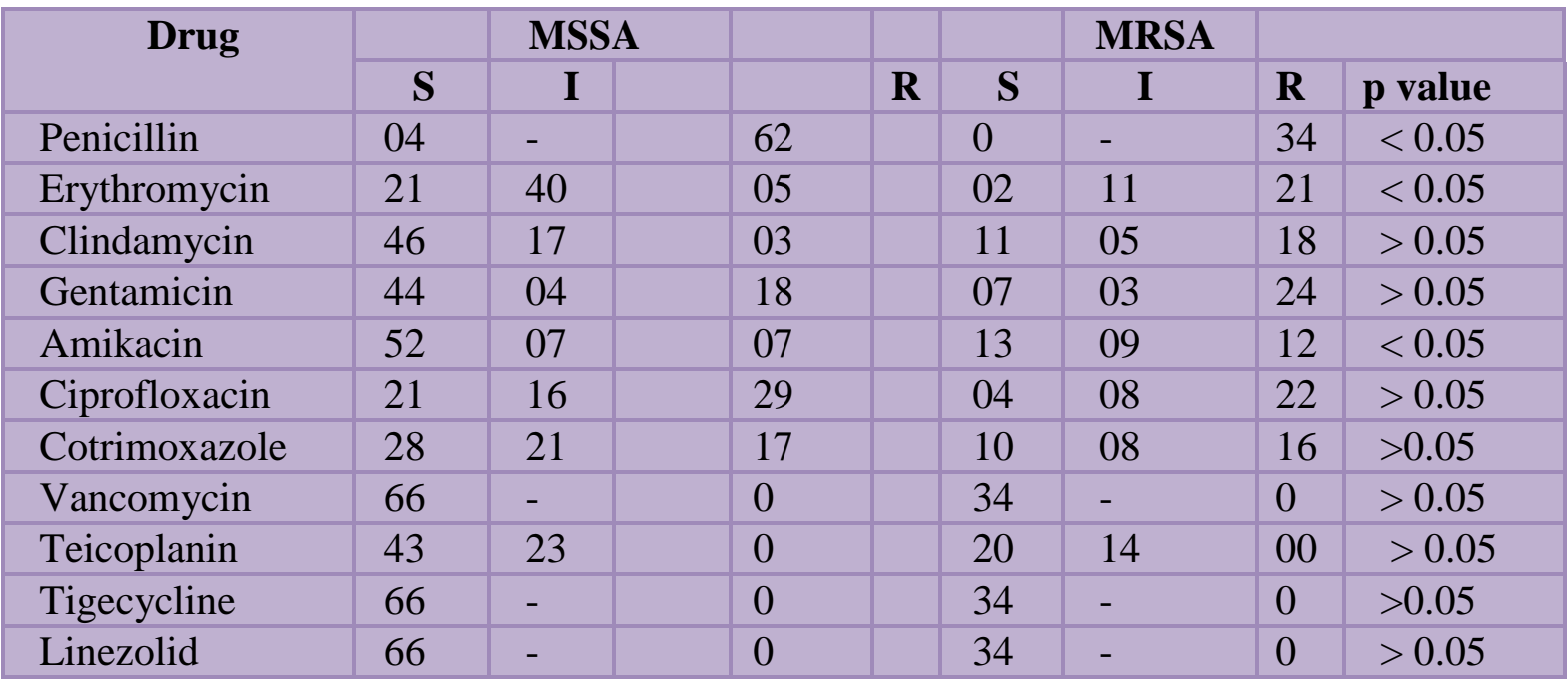

$\mathrm{S}=$ Sensitive, I= Intermediate, $\mathrm{R}=$ Resistant 
Fig.1 Distribution of MRSA isolates by age and gender

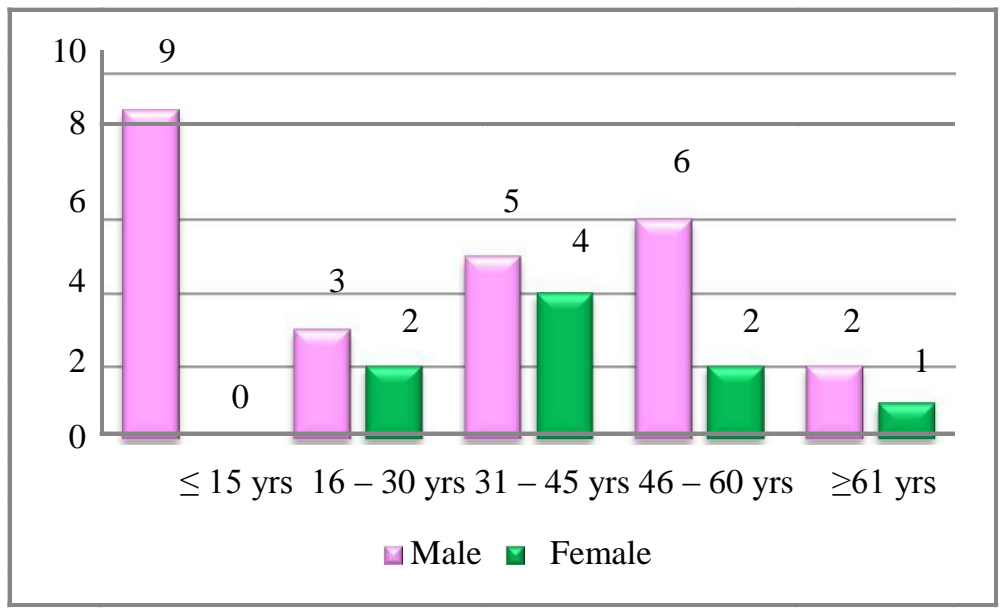

Fig.2 MRSA categorization on infection basis

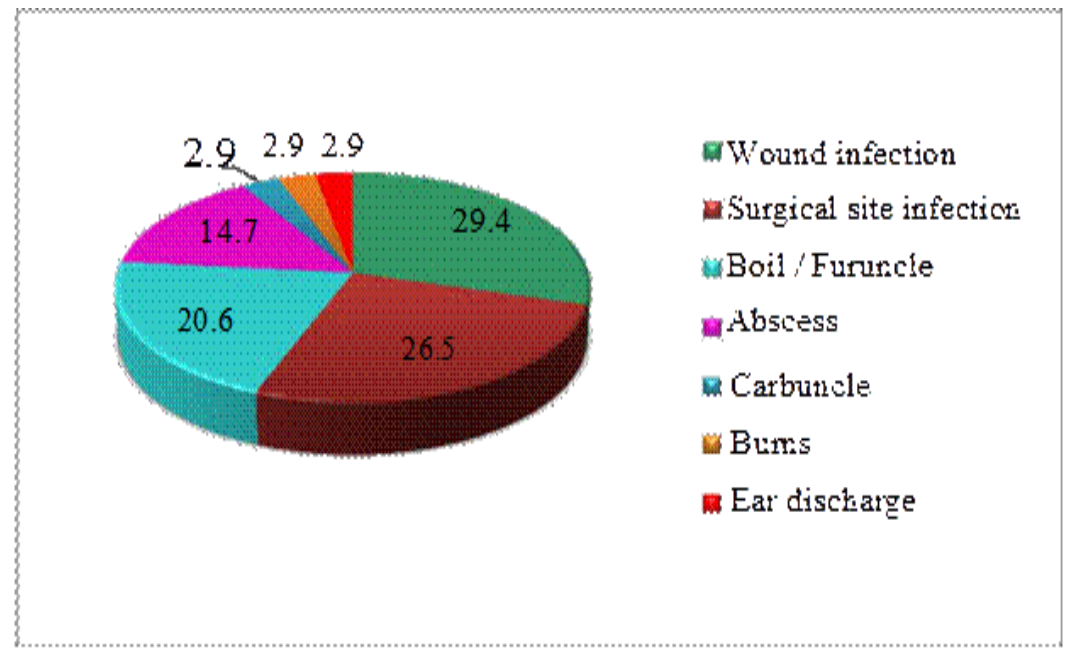

The role of Erythromycin as an alternative among MRSA infections is uncertain because of its higher resistance. Gentamicin resistance was $18(27.3 \%)$ among MSSA isolates and 24 $(70.6 \%)$ among MRSA isolates. Terry Ali et al., ${ }^{11}$ had found $56.5 \%$ resistance and the study from Manglore ${ }^{16}$ found $40-50 \%$ of the MRSA isolates were resistant to Gentamicin. A $100 \%$ resistance was observed among the MRSA isolates in the study from Assam ${ }^{18}$. But the study by Rajaduraipandi et al., ${ }^{17}$ had reported $20.5 \%$ to Gentamicin among MRSA isolates. Among aminoglycosides, the role of
Gentamicin for MRSA infections can be ruled out, as it is frequently administered for Gram negative infections.

In this study, $7(10.6 \%)$ resistance among MSSA isolates and $12(35.3 \%)$ among MRSA isolates were noted for Amikacin. This is similar to the study from Amristar ${ }^{8}$, where the resistance to Amikacin was $12(8.9 \%)$ among MSSA isolates and 43 (37.4\%) among MRSA isolates. This is in opposition to the study by Mullah et al., ${ }^{19}$ where the Amikacin resistance was found to be $52.6 \%$. But a study 
from $\operatorname{Iran}^{20}$, had showed only $13.8 \%$ resistance. Amikacin still remains effective for MRSA infections. Resistance to Ciprofloxacin is considered to be surrogate marker for MRSA infections. In this study, Ciprofloxacin resistance was found to be $64.7 \%$ among MRSA isolates. The study by Rao et al., ${ }^{16}$ found $53-56 \%$ resistance for Ciprofloxacin among MRSA isolates. The study by Pai et al., ${ }^{21}$ found only $31.8 \%$ resistance among MRSA isolates. Quershi et $a l .{ }^{22}$, found higher resistance among MRSA isolates $(90 \%)$ to Ciprofloxacin.

In the present study, multidrug resistance was observed more among MRSA isolates like Erythromycin, Clindamycin, Gentamicin, Amikacin, Ciprofloxacin and Co-trimoxazole. These are considered as the first line treatment for MRSA infections and their resistance is of major concern as treatment goes in favour of glycopeptides, which are the reserve drugs and are also expensive. This can give rise to unexpected outbreaks in hospital.

In conclusion, emergence of antibiotic resistance contributes to significant mortality and morbidity and continues to remain as main hindrance in the control of infectious diseases. The early detection of Methicillin resistance among $S$. aureus together with timely and ideal antibiotic initiation helped by antibiotic sensitivity testing is the need of the hour, which limits the spread of MRSA infections in hospital setting. The role played by a clinical microbiologist in early detection of MRSA is very critical which helps to avoid multidrug resistance, in an era of very limited antibiotics.

\section{References}

1. Cassandra D. Salgado, Barry M. Farr, David P. Calfee. Community-Acquired Methicillin-Resistant Staphylococcus aureus: A Meta-Analysis of Prevalence and Risk Factors. Clinical Infectious Diseases. 2003: 36: 131-9.

2. Humphreys H. Staphylococcus. In: Greenwood D, Slack R, Peutherer J, Barer M. editors. Medical Microbiology. Churchill Livingstone: Elsevier; 2007. Pp. 172-77.

3. Susan S. Huang, Richard Platt. Risk of Methicillin-Resistant Staphylococcus aureus Infection after Previous Infection or Colonization. Clinical Infectious Diseases. 2003; 36:281-5.

4. Clinical and Laboratory Standards Institute. Performance Standards for antimicrobial susceptibility testing; Seventeenth informational supplement. CLSI document M100-S17. Wayne Pa. Clinical and Laboratory Standards Institute. 2007; 27 (1).

5. Thangavel A, Natarajan M, Moses RP. Comparative identification of methicillin resistant Staphylococcus aureus (MRSA) based on amplification of mecA gene and growth on MeReSa agar and Hichrome MeReSa agar. Int $J$ Genetic Engg Biotechnol. 2011; 2: 163-72.

6. Sharma S, Mall A. The Prevalence, antibiogram and characterization of methicillin resistant Staphylococcus aureus among the patients from the Doon valley hospitals. Afr $\mathrm{J}$ microbiol Res. 2011; 5(21): 3446-51.

7. Buzaid N, Elzouki AN, Taher I, Ghenghesh KS. Methicillin-resistant Staphylococcus aureus (MRSA) in a tertiary surgical and trauma hospital in Benghazi, Libya. J Infect Dev Ctries 2011; 5(10): 723-26.

8. Arora S, Devi P, Arora U, Devi B. Prevalence of methicillin-resistant Staphylococcus aureus (MRSA) in a tertiary care hospital in Northern India. J Lab Physici. 2010; 2(2):78-81.

9. Sarma JB, Ahmed GU, Characterisation of methicillin resistant $S$. aureus strains and risk factors for acquisition in a teaching hospital in north east India. Ind J Med Microbiol. 2010; 28(2):127-9. 
10. Sadaka SM, El-Ghazzawy EF, Harfoush RA, Meheissen MA. Evaluation of different methods for the rapid diagnosis of methicillin-resistance in Staphylococcus aureus Afr J Microbiol Res. 2009; 3(2): 49-55.

11. Terry Alli OA, Ogbolu DO, Mustapha JO, Akinbami R, Ajayi AO. The non association of Panton- Valentine leukocidin and mecA genes in the genome of Staphylococcus aureus from hospitals in South Western Nigeria. Ind $\mathbf{J}$ Med Microbiol. 2012; 30(2): 159-64.

12. Srinivasan S, Sheela D, Shashikala, Mathew R, Bazroy J,Kanugo R. Risk factors and associated problems in the management of infections with methicillin resistant Staphylococcus aureus. Ind $\mathrm{J}$ Med Microbiol. 2006; 24(3):182-5.

13. Frazee BW, Lynn J, Charlebois ED, Lambert L, Lowery D, PerdreauRemington F High Prevalence of Methicillin-Resistant Staphylococcus aureus in Emergency department Skin and Soft Tissue Infections. Ann Emer Med. 2005; 45(3):311-20.

14. Haddadin AS, Fappiano SA, Lipsett PA Methicillin resistant Staphylococcus aureus (MRSA) in the intensive care unit.Postgrad Med J. 2002; 78:385-392.

15. Mehta A P, Rodrignes C, Sheth K, Jani S, Hakimiyan A, Fazalbhoy N. Control of methicillin resistant Staphylococcus aureus in a tertiary care centre - A five year study. J Med microbial. 1998; 16:314.

16. Rao VI, Kishore BG, Manohar KS, Vidya P, Manjula S. Detection of Methicillin
Resistance in Staphylococcus aureus: Comparison of Disc diffusion and MIC with mecA gene detection by PCR. Int $\mathrm{J}$ Pharm Bio Sci. 2011; (4): 518-21.

17. Rajaduraipandi K, Mani KR, Panneerselvam K, Mani M, Bhasker M, Manikandan P. Prevalence and antimicrobial susceptibility pattern of methicillin resistant Staphylococcus aureus: A multicentre study. Ind J Med Microbiol. 12006; 24(1):34-8.

18. Majumder D, Bordoloi JNS, Phukan AC, Mahanta J Antimicrobial susceptibility pattern among methicillin resistant staphylococcus isolates in Assam. Ind J Med Microbiol. 2001; 19(3): 138-140.

19. Mulla S, Patel M, Shah L, Vaghela G. Study of antibiotic sensitivity pattern of methicillin-resistant Staphylococcus aureus. Indian J Crit Care Med. 2007;11(2):99-101.

20. Zeinalli E, Moniri R, Musari GH. Antibiotic resistance and molecular subtypes of clinical methicillin-resistant Staphylococcus aureus in a teaching hospital. Ind J Med Microbiol2011; 29(3): 318-9.

21. Vidhani S, Mehndiratta PL, Mathur MD. Study of methicillin resistant $S$. aureus (MRSA) isolates from high risk patients. Ind J Med Microbiol. 2001; 19(2): 13-16

22. Qureshi AH, Rafi S, Qureshi SM, Ali AM. The current susceptibility patterns of methicillin resistant Staphylococcus aureus to conventional anti staphylococcus antimicrobials at Rawalpindi. Pak J Med Sci. 2004; 20: 361-4.

\section{How to cite this article:}

Susitha, T. and Sudha, M. 2019. Risk Factors and Antibiotic Sensitivity Pattern of Staphylococcus aureus Isolates from Suppurative Lesions at a Tertiary Care Hospital. Int.J.Curr.Microbiol.App.Sci. 8(03): 431-437. doi: https://doi.org/10.20546/ijcmas.2019.803.054 